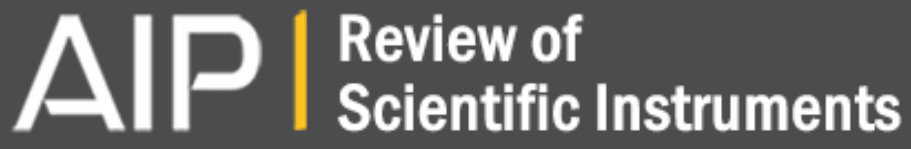

\section{Development of the gas-puff imaging diagnostic in the TEXTOR tokamak}

I. Shesterikov, Y. Xu, M. Berte, P. Dumortier, M. Van Schoor, M. Vergote, B. Schweer, and G. Van Oost

Citation: Review of Scientific Instruments 84, 053501 (2013); doi: 10.1063/1.4803934

View online: http://dx.doi.org/10.1063/1.4803934

View Table of Contents: http://scitation.aip.org/content/aip/journal/rsi/84/5?ver=pdfcov

Published by the AIP Publishing

\section{Articles you may be interested in}

New dual gas puff imaging system with up-down symmetry on experimental advanced superconducting tokamak Rev. Sci. Instrum. 83, 123506 (2012); 10.1063/1.4770122

Comparison of scrape-off layer turbulence simulations with experiments using a synthetic gas puff imaging diagnostic

Phys. Plasmas 18, 022306 (2011); 10.1063/1.3553024

Microwave imaging reflectometer for TEXTOR (invited)

Rev. Sci. Instrum. 74, 1426 (2003); 10.1063/1.1530381

Three-dimensional fluctuation imaging diagnostic for TEXTOR (abstract)

Rev. Sci. Instrum. 72, 363 (2001); 10.1063/1.1323469

Edge turbulence measurements in NSTX by gas puff imaging

Rev. Sci. Instrum. 72, 931 (2001); 10.1063/1.1321009

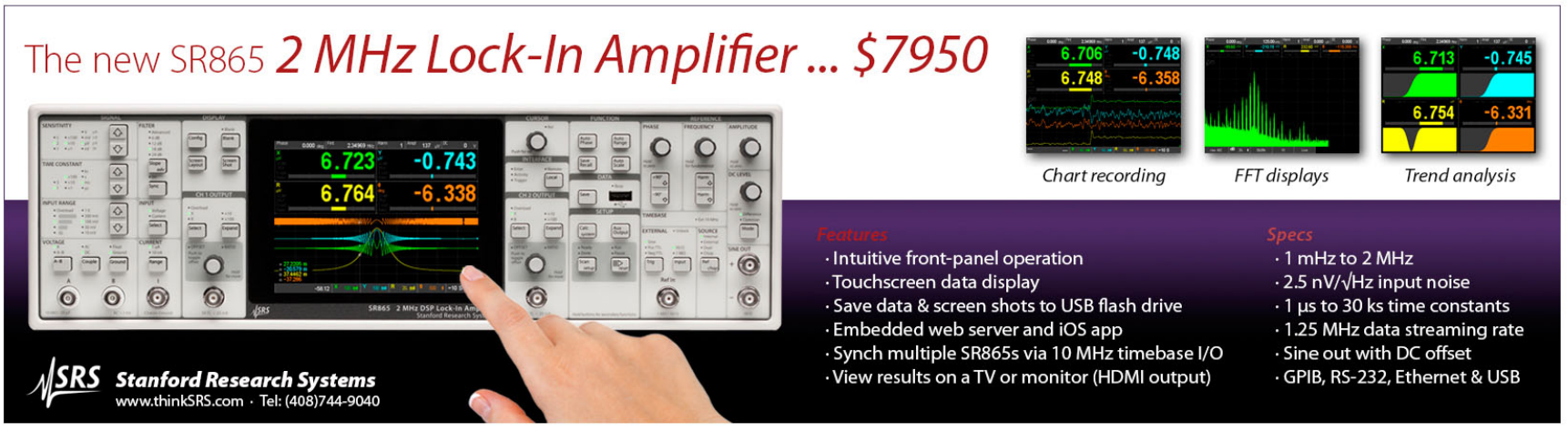




\title{
Development of the gas-puff imaging diagnostic in the TEXTOR tokamak
}

\author{
I. Shesterikov, ${ }^{1}$ Y. Xu, ${ }^{1}$ M. Berte, ${ }^{1}$ P. Dumortier, ${ }^{1}$ M. Van Schoor, ${ }^{1}$ M. Vergote,${ }^{1}$ \\ B. Schweer, ${ }^{2}$ and G. Van Oost ${ }^{3}$ \\ ${ }^{1}$ Laboratoire de Physique des Plasmas - Laboratorium voor Plasmafysica, Association 'Euratom-Belgian \\ state', Ecole Royale Militaire - Koninklijke Militaire School, Trilateral Euregio Cluster, B-1000 Brussels, \\ Belgium \\ ${ }^{2}$ Institut für Energie- und Klimaforschung - Plasmaphysik, Forschungszentrum Jülich GmbH \\ EURATOM-Association, Trilateral Euregio Cluster, D-52425 Jülich, Germany \\ ${ }^{3}$ Department of Applied Physics, Ghent University, B-9000 Ghent, Belgium
}

(Received 15 October 2012; accepted 22 April 2013; published online 9 May 2013)

Gas puff imaging (GPI) [S. J. Zweben, D. P. Stotler et al., Phys. Plasmas 9, 1981 (2002); R. J. Maqueda, G. A. Wurden et al., Rev. Sci. Instrum. 74, 2020 (2003)] is a powerful diagnostic that permits a two-dimensional measurement of turbulence in the edge region of a fusion plasma and is based on the observation of the local emission of a neutral gas, actively puffed into the periphery of the plasma. The developed in-vessel GPI telescope observes the emission from the puffed gas along local (at the puff) magnetic field lines. The GPI telescope is specially designed to operate in severe TEXTOR conditions and can be treated as a prototype for the GPI systems on next generation machines. Also, the gas puff nozzle is designed to have a lower divergence of the gas flow than previous GPI diagnostics. The resulting images show poloidally and radially propagating structures, which are associated with plasma blobs. We demonstrate that the local gas puff does not disturb plasma properties. Our results indicate also that the neutral gas emission intensity is more sensitive to the electron density than the electron temperature. Here, we present implementation details of the GPI system on TEXTOR and discuss some design and diagnostic issues related to the development of GPI systems in general. [http://dx.doi.org/10.1063/1.4803934]

\section{INTRODUCTION}

Turbulence in the edge region of tokamaks and other magnetized plasmas has been studied for many years. The topic is important for the progress of magnetic fusion since edge turbulent transport plays a significant role in both the plasma-wall interaction and the plasma performance. The transport at the edge and the scrape-off layer (SOL), which determine the local temperature and density, depends on the level and properties of turbulence. The edge conditions then affect the core confinement through their effects on edge gradients and gradient driven instabilities such as drift waves. The edge turbulence also plays a crucial role in the improved confinement (H-mode) where edge pedestals in the temperature and density are formed nearby the separatrix.

Turbulent transport in the SOL shows an intermittent blobby behaviour. ${ }^{3,4}$ Blobs are coherent structures extending in a filamentary way along the magnetic field line. They propagate radially and leave the plasma at high speeds. They may increase the recycling in the main chamber and hence result in a high level erosion of the first wall. ${ }^{5,6}$ Therefore, characterization of blobby transport and understanding the underlying physical mechanisms of blob generation and evolution are extremely important topics in fusion research.

Two methods are commonly used for 2D turbulence measurements: Langmuir probe arrays ${ }^{7-9}$ and beam emission spectroscopy (BES). ${ }^{10,11}$ However, BES typically does not have enough spatial resolution and Langmuir probes cannot penetrate very deep into the hot plasma. Gas puff imaging (GPI) presents an interesting alternative as it is not subject to some of the drawbacks of other systems. In the present work, we refer to a GPI system as a system with a observation direction along the magnetic field line, as imposed in pioneering work in Refs. 1 and 2.

Over the past 10 years some essential progress in GPI systems has been made, but so far the diagnostic has only been implemented in diverted tokamaks and linear machines. ${ }^{1,2,12}$ The aim of this paper is to report on the first implementation of a GPI system on the TEXTOR limiter tokamak. We describe the setup details, diagnostic issues, and results obtained. Section II gives brief overview of basic principles that underlie GPI measurements. Some fundamental limitations of the diagnostic will be discussed in Sec. III. The experimental results are presented in Sec. IV.

\section{GPI DIAGNOSTIC}

$\mathrm{GPI}^{1,2}$ is a relatively new tool, which allows to observe the two-dimensional features (poloidal vs radial) of plasma edge turbulence in fusion devices. A neutral deuterium (D) cloud is puffed into the edge plasma region. The visible light emission from the gas cloud is then imaged with a fast framing camera with exposure times (typically of the order of few $\mu \mathrm{s})$, shorter than the autocorrelation time of the turbulence, in order to capture the motion of the turbulent structures.

The GPI system was developed for the TEXTOR tokamak with the major radius $R=175 \mathrm{~cm}$ and minor radius $a=47.5 \mathrm{~cm}$. Typical plasma current in the experiments was $I_{p}=(300-350) \mathrm{kA}$, toroidal magnetic field $B_{T}$ $=(1.6-2.6) \mathrm{T}$ and the central line-averaged electron density $\bar{n}_{e 0}=(1.5-3.5) \times 10^{19} \mathrm{~m}^{-3}$. The volume of the TEXTOR 


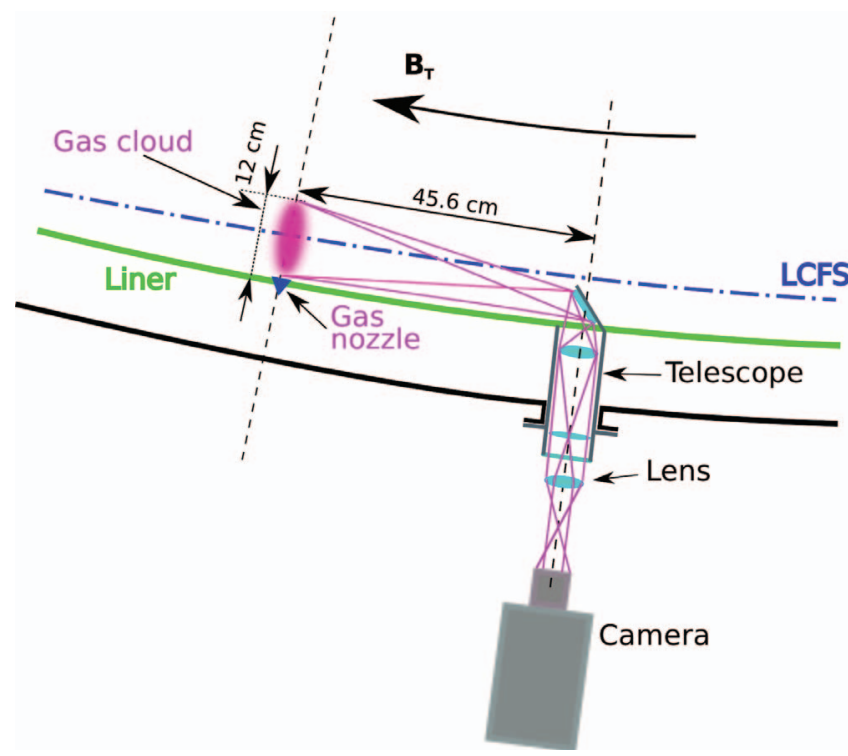

FIG. 1. Schematic illustration (top view) of the GPI system in TEXTOR. The emission from the gas cloud is viewed along the local (at the puff) magnetic field line by an optical telescope, installed in the vessel. The observation covers a view field $12 \mathrm{~cm}$ in diameter in the radial vs poloidal plane from the plasma edge to the wall. The light passes through the telescope's lenses and is guided to the fast frame camera installed about $1.3 \mathrm{~m}$ away from the TEXTOR vessel.

vacuum vessel ${ }^{13}$ is $17.4 \mathrm{~m}^{3}$. A schematic illustration of the GPI geometry (top view) is shown in Fig. 1. A neutral gas cloud is puffed continuously in the vacuum chamber for half a second through the gas inlet nozzle installed on the liner. The emitted light is observed via a telescope, installed on the equatorial plane nearby the nozzle (see also Fig. 2) at a toroidal distance of $45.6 \mathrm{~cm}$. With this configuration we obtain a viewing area $12 \mathrm{~cm}$ in diameter at the cloud plane. The system is adjusted so that the separatrix passes through the middle of the observation area and divides the view field in two approximately equal parts corresponding to edge and SOL plasmas. Therefore, the evolution of turbulent structures, from the birth in the edge to the fast decay in the SOL can be observed.

After passing through the telescope optics, the light is guided to the objective of the fast frame CCD camera, installed $1.3 \mathrm{~m}$ away from the TEXTOR vessel. A $D_{\alpha}$ optical filter $(656 \mathrm{~nm})$, installed in front of the CCD chip, is used to discriminate the brightest deuterium line $(n=3 \rightarrow 2$ transition in the Balmer series).

Figure 2 shows the in-vessel components of the GPI system. The viewing line of the telescope system is along the local (at the gas puff cloud) magnetic field which has a pitch angle $\alpha \approx 2.5^{\circ}$ with respect to the horizontal plane. This value is estimated for $r=47.5 \mathrm{~cm}, I_{p}=250 \mathrm{kA}$ and $B_{T}=2.25 \mathrm{~T}$. Several observations ${ }^{14-16,28}$ indicate that the turbulent structures are highly correlated along the magnetic field lines. Therefore, the dynamics of turbulence in the poloidal vs radial plane seems to be more informative. Consequently, the observation line of sight of the gas puff emission is normally along the magnetic field line at the location of the gas cloud.

The gas injection system is composed of a deuterium molecules reservoir and tubing system which connect the reservoir to the exit nozzle. The nozzle is installed inside the TEXTOR vessel (fixed on the liner) at a radial position (minor radius) $r \approx 53 \mathrm{~cm}(5.5 \mathrm{~cm}$ outside of the last closed flux

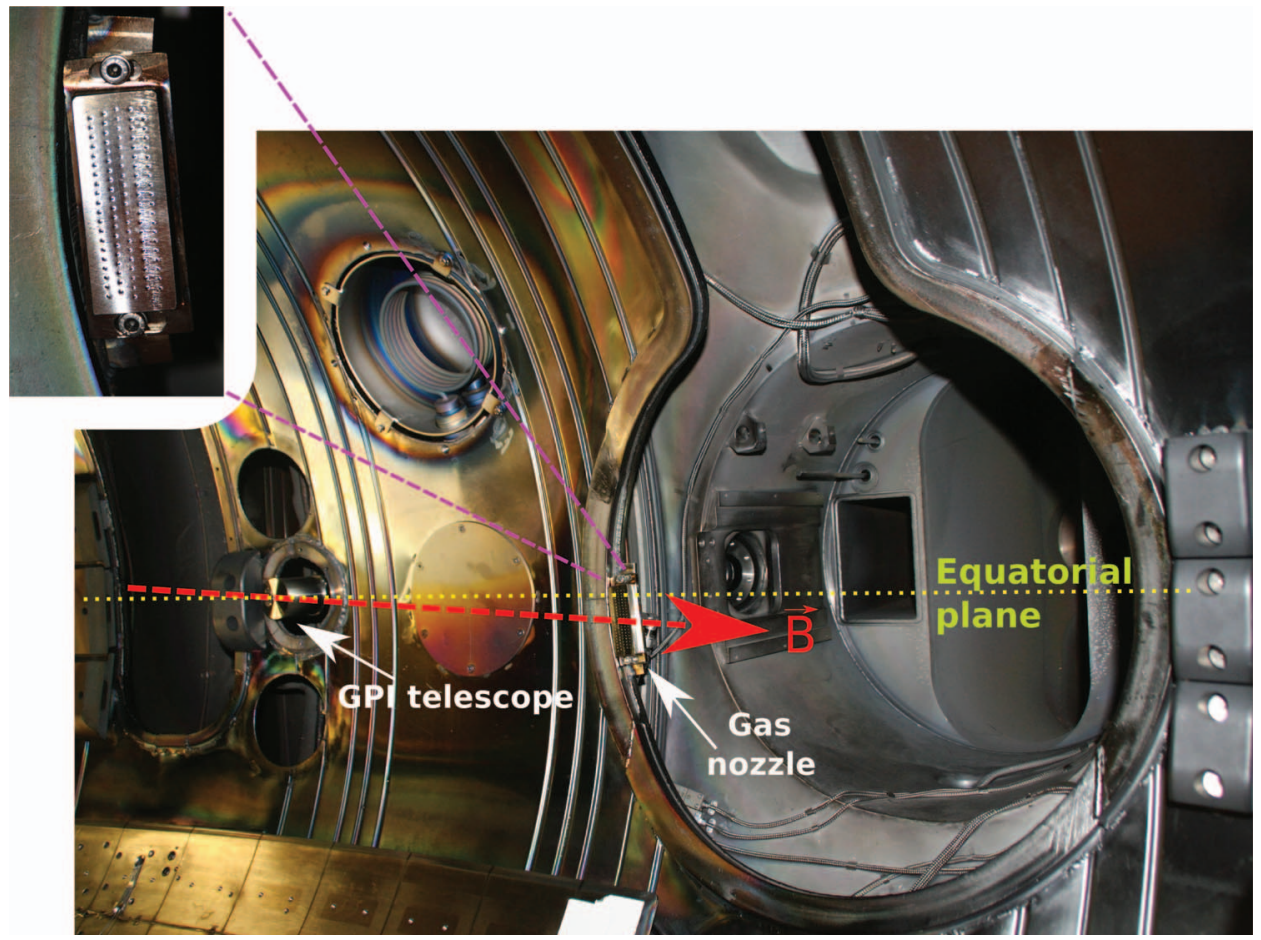

FIG. 2. In-vessel components of the GPI system at TEXTOR. The neutral gas enters the chamber radially via the gas-puff nozzle. Its emission is viewed along the local (at the gas cloud) magnetic field $(\vec{B})$ by a telescope installed in an equatorial port at a distance of $45.6 \mathrm{~cm}$ from the gas inlet nozzle. The inset at the top shows the capillary gas inlet nozzle composed of 100 holes each with a diameter of $0.5 \mathrm{~mm}$. 
surface (LCFS)). The tubing system consists of external and in-vessel tubes. The interface between both tubes (the gas injection entrance port) is a short "Vespel" tube to ensure electrical insulation between the vacuum vessel and the outside hardware. The external tube is $6 \mathrm{~mm}$ inner diameter, $7 \mathrm{~m}$ long stainless steel tube. The in-vessel one is $6 \mathrm{~mm}$ inner diameter, $0.5 \mathrm{~m}$ long stainless steel flexible tube. Flexibility is necessary to remove mechanical tensions in the tube raised by bakeout heating or disruptions.

It is always reasonable to reduce the divergence of the GPI gas puff in order to minimize the plasma disturbance. However, this has not been implemented, for instance, in NSTX and C-Mod tokamaks whereas some other gas-puffing systems such as on LHD and TEXTOR use the supersonictype nozzle. In our experiment, to reduce the divergence of particles, we used "shower-like" multi-capillary source made up of 100 holes mechanically drilled in a rectangular matrix with the size of $2 \times 6 \mathrm{~cm}^{2}$. Each hole has the diameter of $\mathrm{d}=0.5 \mathrm{~mm}$ and the length of $\mathrm{L}=15 \mathrm{~mm}$ (aspect ratio $A=L / d$ $=30$ ). Alternatively, the stack of stainless steel hypodermic needles (or standard capillary tubes available in the industry), laser drilled holes or supersonic de Laval nozzle can be implemented to make beam more unidirectional. However, the practical implementation of these approaches is more costly and time-consuming. Our choice is the compromise between lower divergence of smaller hole and rising fabrication cost of alternative approaches. The plasma facing side of the nozzle is zoomed in the inset of Figure 2. The matrix of holes is drilled in the orthogonal stainless steel bar.

One important quantity, the Knudsen number $(\mathrm{Kn})$, describes the state of the gas flow. Depending on the value of $K n$ we can approach "molecular flow" $(K n \gg 1)$, "transient flow" $(0.01<K n<1)$, or "viscous flow" $(K n<0.01)$ at the nozzle exit. The "viscous flow" is naturally characterized by a wide divergence (which is undesirable for us) of the beam density and this divergence cannot be improved anyhow with any aspect ratio A of the nozzle hole. In this type of flow, the angular dependence of the beam density as a function of the angle $\theta$ to the beam axis is described by so called "cosine law." The divergence of the beam in this regime, measured at full width at half maximum (FWHM), is $\approx 120^{\circ}$. This type of flow is typical, for instance, for the GPI experiment on Alcator C-Mod. The outflow from the nozzle will be as much unidirectional as far we are in the "molecular flow" regime. The divergence in this regime could be just several degrees, depending on the Knudsen number and aspect ratio A of the nozzle hole. The higher $\mathrm{Kn}$ and A values, the lower the divergence. The "transient flow" is characterized by some intermediate divergence. The Knudsen number is defined as the ratio of the molecular mean free path length $\lambda$ to an inner diameter of each hole d:

$$
K n=\lambda / d \text {. }
$$

The mean free path length $\lambda$ is estimated as follows:

$$
\lambda=k_{B} T / \sqrt{2} \pi \sigma^{2} p .
$$

Here, $k_{B}$ is the Boltzmann constant, $T$ is the thermodynamic temperature $(300 \mathrm{~K}), \sigma$ is the molecule hard shell diameter of a deuterium molecule (297 pm), $p$ is the static pressure. Now let us estimate the $K n$ value for our gas outflow through the nozzle. The working pressure in the molecule reservoir is 25 mbar. However, there is a pressure gradient between the molecules reservoir and the nozzle and the pressure just behind the nozzle exit to the vacuum has never been measured. However, the pressure behind the nozzle exit could be straightforwardly obtained from the measured total molecular flux, assuming that the outflow velocity $v$ at the nozzle exit equals to the sound speed $c_{s}=\sqrt{\gamma R T / M_{m}}$,

$$
P=\rho v^{2} / 2=J_{\text {molec }} \sqrt{\gamma R T M_{m}} /\left(2 N_{a}\right),
$$

where $\gamma$ is the adiabatic index (roughly taken for hydrogen $\sim 1.4$ ), $J_{\text {molec }}$ is the gas flux density, $R$ is the universal gas constant, $M_{m}$ is the molar mass of the deuterium in kilograms per mole $\left(4.028 \times 10^{-3} \mathrm{~kg} / \mathrm{mol}\right), N_{a}$ is the Avogadro constant. The gas flux density $J_{\text {molec }}$ for each nozzle hole is the following:

$$
J_{\text {molec }}=4 F /\left(N \pi d^{2}\right) .
$$

Here, $F$ is the total gas flow through the nozzle, $N$ is the total number of capillary holes in the nozzle (100). For the total flux of $F=1.2 \times 10^{20}$ molecules/s, the flux density for each hole is $J_{\text {molec }}=6.1 \times 10^{24}$ molecules $/\left(\mathrm{s} \mathrm{m}^{2}\right)$ and the pressure behind the nozzle exit is $P_{d}=0.36 \mathrm{mbar}$. The corresponding Knudsen number is $K n \approx 0.57$. This indicates that the practical gas outflow is in the "transient flow" regime and we can expect some improvements in the divergence with respect to the previous GPI experiments.

An experiment has been performed to measure the angular profile of the gas flow from the nozzle using the quadrupole mass spectrometer. The intensity of the gas beam has been spatially scanned along a linear path $\zeta$ intersecting the gas forming hole axis in the perpendicular direction. The measurements have been done in the separate test vacuum chamber at the distance of $h \sim 20 \mathrm{~cm}$ from the nozzle exit. Note that our nozzle is not the point source and instead has a finite extension along the direction of the scan. The scan direction is selected along the narrow side $(2 \mathrm{~cm})$ of the nozzle exit plane. At a distance of $20 \mathrm{~cm}$ from the nozzle exit plane, we can ignore $2 \mathrm{~cm}$ size of the nozzle and approximately represent it as a point source. The linear path $\zeta$ covers the distance of $\zeta \in[-10 \ldots 10] \mathrm{cm}$ with respect to the center of the nozzle exit plane. When studying the divergence of the beam, the associated physics might be more transparent if one presents the beam density $I^{\text {beam }}$ in the polar coordinate system as a function in terms of an angle $\theta$ with respect to the beam axis, i.e., $I^{\text {beam }}(\theta)$. Therefore, the intensity distribution along linear path has been converted to polar coordinates using the relation $\theta=\arctan (\zeta / h)$. The results are shown in Fig. 3. The green line shows the normalized beam density $I^{\text {beam }}(\theta) / I^{\text {beam }}(0)$ as a function of the angle $\theta$. The distribution is shown for the typical working gas pressure in the reservoir of 25 mbar. The cosine law distribution, typical, for instance, for the viscous outflow of NSTX or C-Mod GPI systems, is shown for comparison by red line. An experiment has shown that the angular profile of the flow from our nozzle has a FWHM at around $20^{\circ}$ and is significantly reduced with respect to the former GPI experiments. That is the essential improvement of the traditional GPI design. Having the source with reduced divergence, the typical deuterium influx 


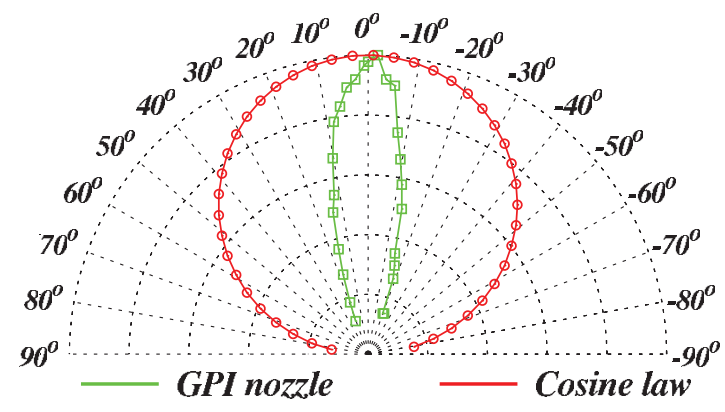

FIG. 3. (green line) The representative measurements of normalized beam intensity $I^{\text {beam }}(\theta) / I^{\text {beam }}(0)$ in polar coordinates as a function of angle $\theta$ with respect to the beam axis, showing the characteristic beam shape. (red line) The cosine law distribution typical for the aperture source (hole in thin plate).

of $7 \times 10^{19}$ molecules/s is used, what is an order of magnitude smaller than that value for NSTX. ${ }^{2}$

Another novel feature of the GPI system on TEXTOR is the optical telescope system, which is installed inside the vessel and sustain severe thermal load and coating. It should be noticed that the in-vessel telescope has not yet been implemented in previous implementations of the GPI system. In Alcator C-Mod, the simple GPI telescope has been used together with in-vessel fiberoptic bundle, whereas the latter can be gradually contaminated by heat load in hot plasmas and neutron flux. Figures 4(a) and 4(b) show the assembly of the optical telescope. From right to left we indicate main components of the telescope: the mirror, the shutter, lenses, the rotary feedthrough, and the glass window. All in-vessel lenses are held in telescope by fluoroplastic rings with the radial thickness of $1 \mathrm{~mm}$ to avoid cracking due to the thermal expansion. The telescope works according to the next description: the light emitted by the gas cloud (marked with yellow arrows) is collected by the mirror and focused with several lenses. After lenses, the light goes through the glass window towards the fast frame camera (not shown in the figure) installed about $1.3 \mathrm{~m}$ away from the TEXTOR flange. The most internal part, the mirror, undergoes a severe transient thermal load and particle flux on its surface. Therefore, it is made as polished $0.5 \mathrm{~mm}$ thick stainless steel plate. To prevent the coating on the telescope mirror, a protective shutter has been installed on the telescope head as also shown in Figure 4 . The rotary feedthrough, bevel gear, and torsional pivot are installed to handle the position of the shutter. The shutter is connected to the torsional pivot and linearly moves along the telescope axis when the pivot rotates. A torque is transmitted from rotary feedthrough to the pivot via the bevel gear. While rotating the feedthrough, the shutter can be moved in the position either to cover and protect the mirror or open it for measurements. The mirror is shuttered off during the glow discharge cleaning, boronization and other experiments when coatings can be deposited on the mirror surface.

The optical design of the system has been performed in optical design software, which provides complete information
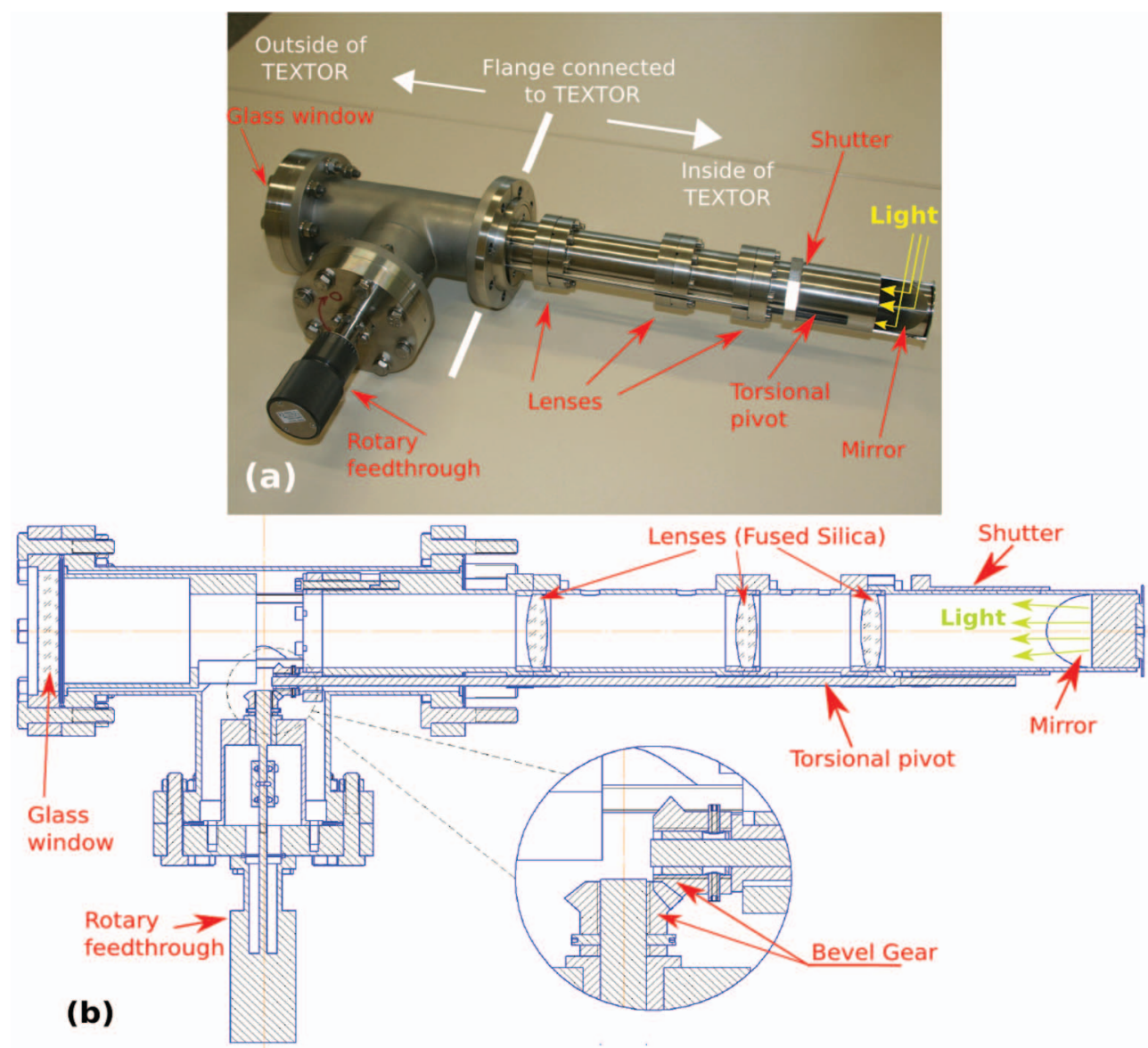

FIG. 4. (a) The photo of the GPI telescope. (b) The section drawing of the GPI telescope system sketching its internal design. 
about aberrations, spot sizes, and quality of the designed optics in general. A PSI-5 fast-frame camera ${ }^{2}$ with a $64 \times 64$ pixel CCD detector has been used with exposure times from 2 to $6 \mu \mathrm{s}$, chosen in function of the plasma parameters (higher plasma densities lead to higher emission and thus to lower exposure time). We do not use a fiber bundle to connect the camera and the telescope to avoid as much as possible loss of light and aberrations in the final image.

All in-vessel lenses are ready-made commercial lenses made of fused silica to sustain high in-vessel temperatures. The high melting point $\left(1723{ }^{\circ} \mathrm{C}\right)$ and low thermal expansion coefficients of fused silica account for its ability to sustain high temperatures (applied, for instance, for the TEXTOR wall conditioning $250-600{ }^{\circ} \mathrm{C}$ ) and rapid temperature changes, caused by disruptions or runaway electrons. The latter two events might significantly rise the temperature up to the melting point. The maximum working temperatures of fused silica is $1000{ }^{\circ} \mathrm{C}$. Although there are several glass types with even higher working temperatures (maximum working temperature of sapphire lenses up to $2000{ }^{\circ} \mathrm{C}$ ), the choice of the fused silica is the compromise between the wide availability of standard lenses made of this material and its heat resistance. Also, all in-vessel lenses are uncoated to avoid the degradation of coatings in high temperature conditions. The optical transmission of the system is $\sim 50 \%$.

Several technical aspects contribute to the optical resolution of the system: optical aberrations, the misalignment of the line of sight with respect to the local magnetic field line direction, number of pixels in the camera sensor. Let us discuss their contribution one by one.

The maximum relative distortion (obtained from the distortion plot of optical design software) of the optical system is $0.07 \%$. Projecting this value onto the object plane and taking into account the diameter of the observation view $\left(L_{o b j}=12 \mathrm{~cm}\right)$ gives the absolute deformation of the measured object of $0.08 \mathrm{~mm}$. The maximum ray spot radius of the optics is $S_{s p}=0.15 \mathrm{~mm}$ in the periphery of the image plane and it is much smaller near the center. This is also obtained from the spot diagrams and transverse ray aberration plots of the design software. Taking into account the magnification factor $\mathrm{M}$ of the system gives the geometric blur size in the object plane. The optical magnification factor $\mathrm{M}$, as the ratio between the imaged size of the object (equals to the camera CCD chip size $L_{i m g}=18 \mathrm{~mm}$ ) and its real size in the object plane $\left(L_{o b j}\right)$, has been obtained $M=L_{i m g} / L_{o b j}=18 / 120=0.15$. Therefore, the contribution of the ray spot radius in the spatial resolution of the optical system is $R_{a b b}=S_{s p} / M=1 \mathrm{~mm}$.

Another issue, relevant to the spatial resolution, is the misalignment of the observation view with respect to the magnetic field line direction due to finite viewing angle of the telescope (in our case $\alpha \approx L_{\text {obj }} / L_{\text {cloud }} \approx 15.2^{\circ}$ ). When the line of sight is not parallel to the field line direction, this causes the loss of the poloidal resolution when observing the turbulent structures which lie along this field line. This degradation in the resolution is bigger the larger the neutral gas cloud is in the line of sight direction. In the TEXTOR tokamak, the extension of the gas cloud along the magnetic field line direction is $\sim 5 \mathrm{~cm}$. For various combinations of $\mathrm{q}$ values in TEXTOR, the pitch angle at the gas cloud varies in the range of $2.3^{\circ}-$ $4.2^{\circ}$, which corresponds to the poloidal resolution $5 \mathrm{~mm}$ $10 \mathrm{~mm}$ within the view field of our optics. On the other hand, as long as the pitch angle remains close to the $2.5^{\circ}$, from which the gas cloud is observed, the poloidal resolution due to misalignment linearly grows from 0 in the center of the view field to $5 \mathrm{~mm}$ on its edge. In the majority of our GPI experiments the pitch angle is within $14 \%$ of the typical value.

Another contribution to the spatial resolution is the pixel number in the camera sensor (only 64 in each direction). This corresponds to the spatial resolution of $R_{c a m}=L_{o b j} / 64$ $\approx 2.0 \mathrm{~mm}$, which is even higher than the resolution defined by the optical aberrations $R_{c a m}>R_{a b b}$. This value cannot be improved anyhow unless another CCD camera is used. Therefore, the total optical resolution is determined mainly by low pixel number in the CCD sensor and it gradually degrades from $2 \mathrm{~mm}$ (dictated by low pixel number) in the center of the view field to $5 \mathrm{~mm}$ on its edge (dictated by non parallel line of sight).

Looking further forward, we are meant to use more advanced CCD cameras with higher number of pixels. In that case, the low number of pixels will not be the factor limiting the spatial resolution of the system anymore and, instead, the resolution will be determined by optical aberrations $\left(R_{a b b}\right)$. Therefore, it is reasonable to discuss some aspects which limit $R_{a b b}$. Optical aberrations could be significantly reduced increasing the distance between the telescope and the gas cloud ( $L_{\text {cloud }} \approx 45 \mathrm{~cm}$ ), thus reducing the angular extent of a imaged cloud and hence approaching the paraxial optics. This measure, however, leads to reduction of the numerical aperture (NA) of the system and hence its light-gathering ability. We refer to the numerical aperture as an object space numerical aperture. The NA value of the system is equal to 0.031 . The actual price paid for the reduction in the amount of light is the enhanced gas puff flux and hence enhanced perturbation of the background plasma, which is not desired in all cases. The NA value would be also higher if we used larger diameter lenses. However, we are limited by the inner diameter of the TEXTOR flange (CF63) and, therefore, all optical components of our telescope have diameter of $40 \mathrm{~mm}$. The distance between the gas cloud and the telescope mirror has been chosen as the compromise between low aberrations (better resolution) of the distant imaging and reduction of the NA (ability to gather light) with increasing distance.

The kinetics of the population of atomic levels for typical edge plasma parameters is described by collisional-radiative (CR) models. ${ }^{17,18}$ The basic assumption of the CR model is that states above ground state will decay much more rapidly than the ground state will change. Based on this, the emission intensity $\epsilon$ from the gas cloud is proportional to the groundstate density $n_{0}$ (i.e., the local density of neutrals in the gas cloud) and, in general, a nonlinear function of the electron density $n_{e}$ and temperature $T_{e}$ :

$$
\epsilon\left(\text { photons } / \mathrm{m}^{3}\right)=n_{0} f\left(n_{e}, T_{e}\right) A,
$$

where $\mathrm{A}$ is the radiative decay rate from the excited electron states (Einstein coefficient) and $f\left(n_{e}, T_{e}\right)$ is a nonlinear function of $T_{e}$ and $n_{e}$. Therefore, the light emission from the plasma is modulated by both the local $T_{e}$ and $n_{e}$ fluctuations. 


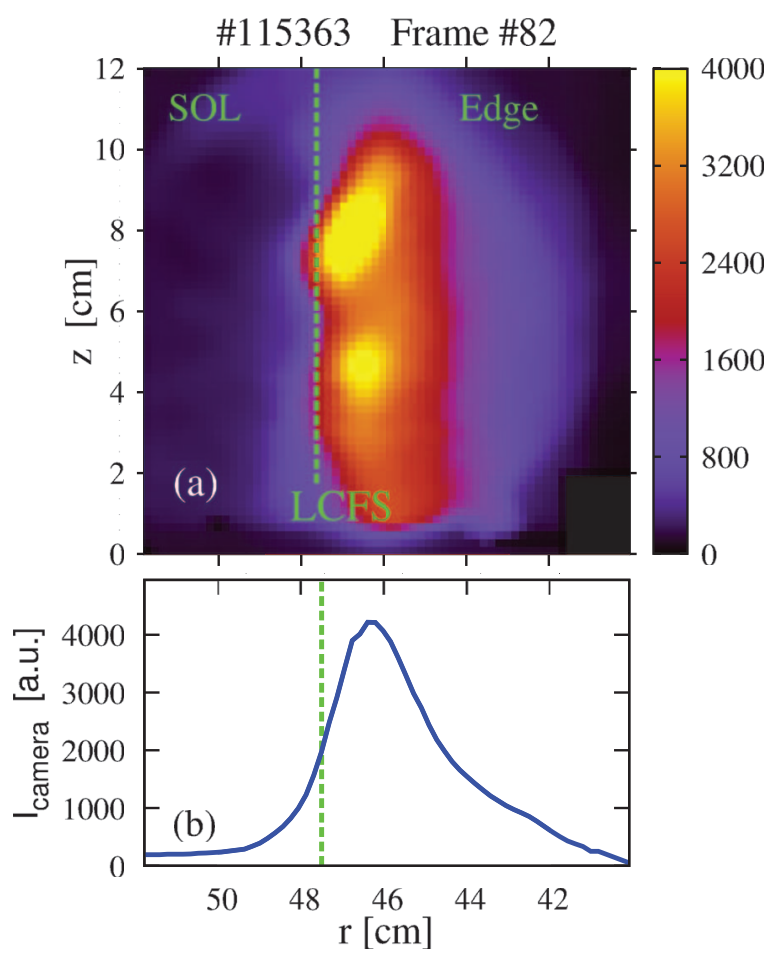

FIG. 5. (a) A single frame of the GPI showing the $D_{\alpha}$ light emission from the neutral deuterium cloud viewed by the optical telescope. The viewing area of the telescope covers $\approx 12 \mathrm{~cm} \times 12 \mathrm{~cm}$ in the poloidal ( $\mathrm{z}$ ) vs radial plane (r). The frame is taken at an exposure time of $6 \mu \mathrm{s}$. The separatrix position is shown by the dashed green line. (b) The 300 -frames averaged radial profile of the $D_{\alpha}$ emission measured with the GPI.

The CR modelling of a molecular deuterium is very complex due to many atomic processes such as dissociation, ionisation, excitation, dissociative excitation, and others. Therefore, the modelling of these processes is beyond the scope of this paper. In this work, we used the typical molecular processes proposed in Refs. 19-23, in which two types of dissociation reactions have been used to explain the production of the atomic deuterium:

$$
\begin{array}{ll}
D_{2}+e^{-} \rightarrow D^{*}(n=3,4,5)+D(1 s)+e^{-} & \text {type }(i), \\
D_{2}+e^{-} \rightarrow D_{2}^{+}+e^{-} & \text {type }(i i) .
\end{array}
$$

The type (ii) reaction requires the subsequent collisional dissociation to produce one deuterium atom:

$$
D_{2}^{+}+e^{-} \rightarrow D^{+}+D^{*}(n=3,4,5)+e^{-} .
$$

Meanwhile the ground atoms can be excited again:

$$
D+e^{-} \rightarrow D^{*}(n=2,3,4,5 \ldots)+e^{-} .
$$

The produced deuterium atoms or other excited ones from $D(1 s)$ after decay will produce Lyman (transition to $n=1$ ) and Balmer (transition to $n=2$ ) series. Among them, transitions from $n=3 \rightarrow 2$ contribute to the $D_{\alpha}$ signal.

Figure 5(a) shows a typical original frame from the GPI system measured with an exposure time of $6 \mu \mathrm{s}$. This image was made in a deuterium cloud and corresponds to an ohmic discharge with $\bar{n}_{e 0}=3.5 \times 10^{19} \mathrm{~m}^{-3}, I_{p}=350 \mathrm{kA}$ and $B_{T}=2.6 \mathrm{~T}$. The artificial color scale (right) has been used to effectively recolor the original camera image. The $\mathrm{x}$ axis corresponds to the radial range of the viewing area in terms of the TEXTOR minor radius. The position of the separatrix is shown in the figure along with the poloidal extension (z) of the view field. The LCFS radial position in the GPI frame is deduced from the radial calibration of the GPI optics while the system was installed at TEXTOR. The cmscale yellow color structures in this image reflect the plasma turbulent eddies. Figure 5(b) shows the 300-frames averaged radial profile of the $D_{\alpha}$ emission intensity $\left(I_{\text {camera }}\right)$ measured in the same shot as in Figure 5(a). Here, in order to understand qualitatively the emission profile one may simply apply the coronal model ${ }^{17,24}$ to roughly analyse the radial dependence of the emission intensity shown in Figure 5(b). According to this model the $D_{\alpha}$ emission intensity is given by

$$
\epsilon(r) \sim n_{e}(r) n_{0}(r)\left\langle\sigma_{1 \rightarrow 3} v_{e}\right\rangle
$$

with $\left\langle\sigma_{1 \rightarrow 3} v_{e}\right\rangle$ denoting the excitation rate from the ground state of a deuterium atom. The line emission $\epsilon(r)$ is monotonically increasing function of the local electron density $n_{e}$ and temperature $T_{e}$. However, the neutral density $n_{0}$ is attenuated towards the centrum by ionization of molecules and atoms on account of electron collisions:

$$
v_{0} \frac{\partial n_{0}}{\partial r}=n_{e}(r) n_{0}(r)\left\langle\sigma_{i} v_{e}\right\rangle,
$$

where $\left\langle\sigma_{i} v_{e}\right\rangle$ is the cross-section of ionisation from the ground state by electron collisions and $v_{0}$ is the velocity of injected atoms. Therefore, two quantities $n_{e}$ and $n_{0}$ included in the expression for $\epsilon(r)$ have opposite radial dependency, resulting in the peaked $\epsilon(r)$ profile as shown in Fig. 5(b). It is clear that the GPI can only be applied in the vicinity of the separatrix.

We have to notice that the GPI-measured emission intensity in Figure 5(b) qualitatively agrees with the measured $D_{\alpha}$ light emission profile in C-Mod ${ }^{1}$ as well as with the results from DEGAS 2 simulations on that machine. In particular, their widths (FWHM) are $2.5 \mathrm{~cm}$ at TEXTOR and $2 \mathrm{~cm}$ at C-Mod and they are both localised in the near vicinity of the separatrix: $1.5 \mathrm{~cm}$ deeper than LCFS at TEXTOR and $0.5 \mathrm{~cm}$ farther at C-Mod. This result is not so obvious because mean time-averaged $I_{\text {camera }}(r)$ profile is defined mainly by the edge $T_{e}(r)$ and $n_{e}(r)$ profiles and despite of similar $T_{e}(r)$ radial profile on both machines (typical values are $20-30 \mathrm{eV}$ on TEXTOR and 20-30 eV on C-Mod), the corresponding $n_{e}(r)$ profiles differ from each other by approximately one order $\left(2.5 \times 10^{-12} \mathrm{~cm}^{-3}\right.$ on TEXTOR and $3 \times 10^{-13} \mathrm{~cm}^{-3}$ on C-Mod). This results might be explained in the context of a simple model mentioned above. Let us assume that the whole $n_{e}(r)$ profile increased in several times. The collisional excitations are more intense the higher the $n_{e}(r)$ values are, according to Eq. (6). On the other hand, the ionization frequency also grows with $n_{e}(r)$ making neutral particles out of the game, according to Eq. (7). Therefore, increased $n_{e}(r)$ profile results in the $I_{\text {camera }}(r)$ profile shifted radially outside whereas the width of the profile might be the same. In other words, all collisional processes enter into the game earlier along the track of particles.

Note, however, that turbulent structures do not affect the whole $n_{0}(r)$ profile. At TEXTOR, the typical radial and poloidal sizes of turbulent structures in the plasma edge is 
$\sim 2 \mathrm{~cm}$, as one can, for instance, see in Fig. 5(a). The typical level of edge plasma density fluctuations, relative to the mean time-averaged value, is $\sim(20-30) \%$. The local $20 \%$ increase in $n_{e}$ does not change significantly $n_{0}$ because $n_{0}$ and $n_{e}$ are related through the differential equation. However, it will rise the emission intensity by $20 \%$, according to linear relation in Eq. (6). Therefore, turbulent structures do not affect significantly $n_{0}(r)$ profile and modulate the emission intensity only locally.

\section{LIMITATIONS OF THE DIAGNOSTIC}

One key requirement for a GPI system is that the gas puff does not affect the local and global plasma parameters neither the turbulence properties. Meanwhile, it should create sufficient emission. To investigate the impact of the gas puff on the local plasma parameters we used a fast reciprocating Langmuir probe mounted at the midplane on the low field side of TEXTOR,${ }^{25}$ toroidally $22.5^{\circ}(86 \mathrm{~cm})$ away from the GPI gas nozzle. Due to this small toroidal distance, the local perturbation of the gas puffing on equilibrium plasma parameters can be seen by the probe as well.

In Figure 6, we present results for an ohmic discharge where we plunge the fast probe twice within one shot: without (blue line) and with the gas puff (red line). The left column shows the results for a gas flux of $1.2 \times 10^{20}$ molecules $/ \mathrm{s}$, while the right column corresponds to $2.3 \times 10^{20}$ molecules $/ \mathrm{s}$.

Figures 6(a) and 6(b) depict the equilibrium electron density for a radial range of $49.8 \mathrm{~cm}>\rho>45.5 \mathrm{~cm}$, including the SOL and the plasma edge. There is no visible difference between the results obtained with and without gas puffing, even for the high flux in Fig. 6(b). The same is valid
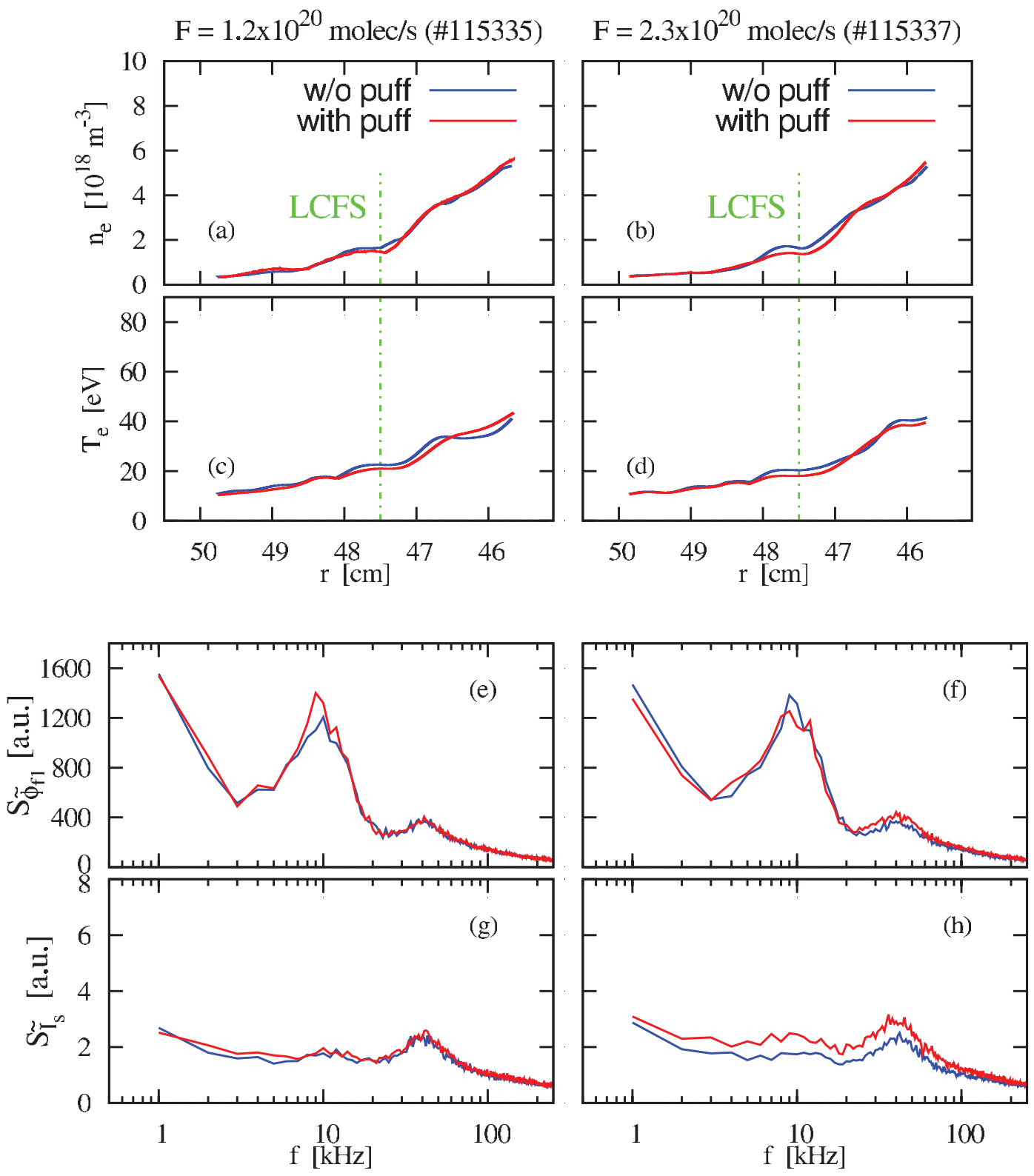

FIG. 6. (a)-(d) Radial profiles of electron density and temperature measured by a Langmuir probe with (blue) and without (red) local gas puffing. (e)-(h) Power spectra of the floating potential fluctuations $\left(S_{\tilde{\phi}_{f l}}\right)$ and ion saturation current fluctuations $\left(S_{\tilde{I}_{s}}\right)$. The left column shows results for a gas flux of $1.2 \times 10^{20}$ molecules/s while the right column for $2.3 \times 10^{20}$ molecules/s. 


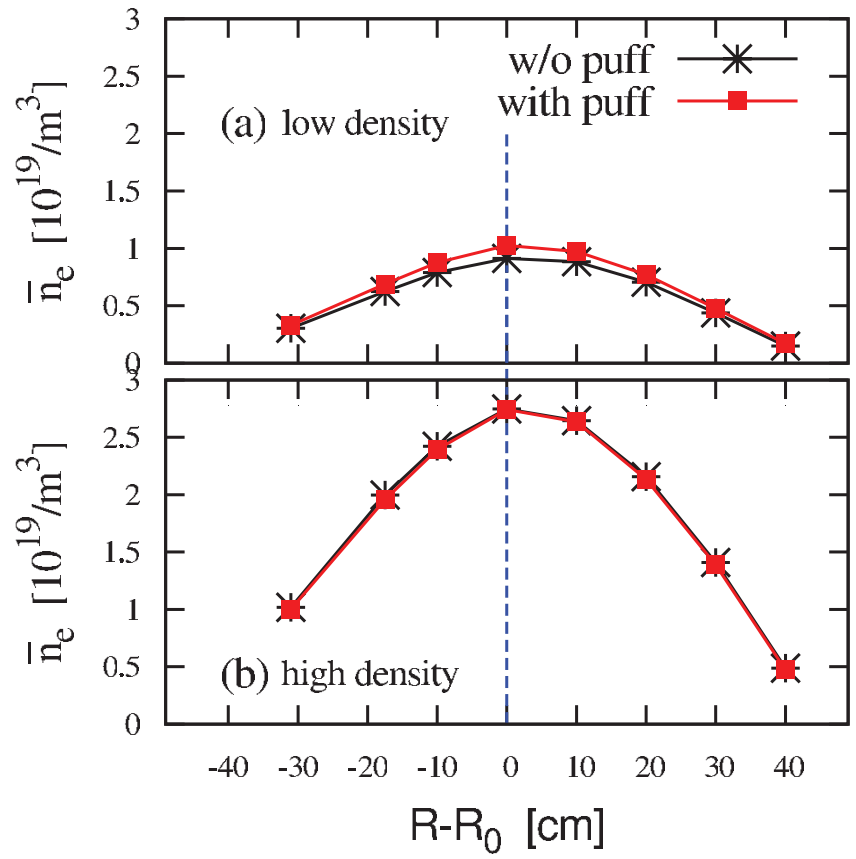

FIG. 7. Comparison of the line-averaged density $\bar{n}_{e}$ obtained with and without the gas injection (the puffing flux is $1.0 \times 10^{20}$ molecules/s). The results of this figure show that the low density shot can be easily perturbed by the gas puffing than the high density shots. Consequently, the working gas puff flux level for all range of plasma densities should be as low as $1.0 \times 10^{20}$ molecules/s.

for the equilibrium electron temperature as can be seen in Figures 6(c) and 6(d), where no visible local cooling of the plasma is observed due to energy losses on dissociation, ionization, and excitation processes. To check whether the edge turbulence is affected, we compared the frequency spectra of $\tilde{\phi}_{f l}$ and $\tilde{I}_{s}$. The FFT spectra of $\tilde{\phi}_{f l}$ for two puffing flux values are compared in Figures 6(e) and 6(f) and show little difference. However, whereas the spectra $S_{\tilde{I}_{s}}$ in Fig. 6(g) show little difference in low puffing case, for high puff flux the spectrum of $\tilde{I}_{s}$ in Fig. 6(h) is clearly affected. From this we conclude that on TEXTOR the GPI system is more suitable to be operated in low gas puff flux level in order to minimize perturbations to local turbulence properties.

In order to survey the influence of the gas puff on the global plasma parameters, we compared the line-averaged densities $\bar{n}_{e}$ without (black lines) and with gas puffing (red lines). The results are shown in Figure 7. In all cases the puffing flux is $1.0 \times 10^{20}$ molecules/s. In low density shots the $\bar{n}_{e}$ profiles show clear difference with and without gas puffing, while in the high density discharges the difference in $\bar{n}_{e}$ is almost not visible. Thus, low density shots are more sensitive to perturbations from the GPI gas injection. This might be explained as follows: at TEXTOR the central line-averaged density $\bar{n}_{e 0}$ is feedback controlled by the internal TEXTOR gas inlet system. In low density shots $\left(\bar{n}_{e 0}=1.0 \times 10^{19} \mathrm{~m}^{-3}\right)$ (see Fig. 7(a)) the TEXTOR internal gas inlet system supplies continuously around $7 \times 10^{19}$ molecules/s in order to stabilize the plasma density. High density shots $\left(\bar{n}_{e 0}=3.0\right.$ $\times 10^{19} \mathrm{~m}^{-3}$ ) $($ see Fig. $7(\mathrm{~b}))$ need around $(8-9) \times 10^{19}$ molecules/s to remain stationary. Therefore, the GPI gas-puff and the TEXTOR gas-flow are comparable and the estab-

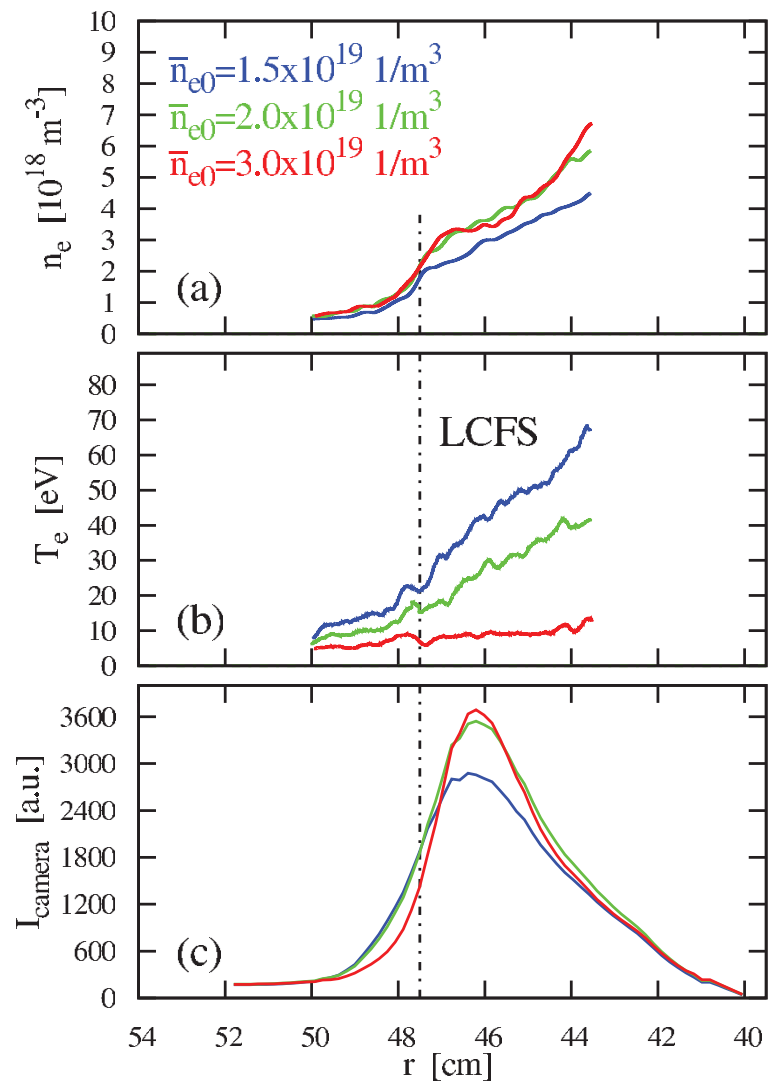

FIG. 8. Comparison of the equilibrium radial profiles of (a) electron density $n_{e}$, (b) electron temperature $T_{e}$, and (c) the 300-frames averaged radial profile of the $D_{\alpha}$ emission measured with the GPI. Each figure shows three profiles obtained for shots with different central line-averaged densities.

lished line-averaged density is the result of the competition between the two systems. In high density shots, the GPI gas inlet system simply replaces the TEXTOR gas inlet system so that there is no net effect. In low density shots, the lower internal TEXTOR gas flow rate is overshadowed by the GPI gas flow leading to a visible increase in the line-averaged density. We should therefore limit the puffing flux to $7 \times 10^{19}$ molecules/s. In practice, we operate in the range of $6 \times 10^{19}$ molecules/s to $8 \times 10^{19}$ molecules/s, depending on the plasma parameters.

We now come to the discussion of the dependence of the emission intensity of the gas cloud on the local plasma parameters. Figure 8 compares the equilibrium radial profiles of (a) electron density $n_{e}$, (b) electron temperature $T_{e}$ (measured with the fast reciprocating Langmuir probe), and (c) the 300 -frames averaged $D_{\alpha}$ emission intensity $I_{\text {camera }}$ (measured with the GPI), corresponding to three shots with different central line-averaged densities $\bar{n}_{e 0}=1.5 \times 10^{19}, 2 \times 10^{19}$, $3.0 \times 10^{19} \mathrm{~m}^{-3}$ ( $I_{p}$ and $B_{T}$ are the same). The $D_{2}$ puff flows for all three shots are the same and, due to $I_{\text {camera }} \propto n_{0}$, we can directly compare $I_{\text {camera }}$ for different plasmas. It is important to note that, while Figure 8(b) shows apparent dependence of the $T_{e}$ profile on the $\bar{n}_{e 0}$, both $n_{e}$ and $D_{\alpha}$ emission profiles are less sensitive to variations of $\bar{n}_{e 0}$. This result suggests that their dependency is rather strong than the dependence of the $I_{\text {camera }}$ on $T_{e}$ and provides support in favor of the principle that the GPI is a diagnostic that measures density rather than temperature events. Also, as seen in Fig. 8(c), the $I_{\text {camera }}$ is higher in 

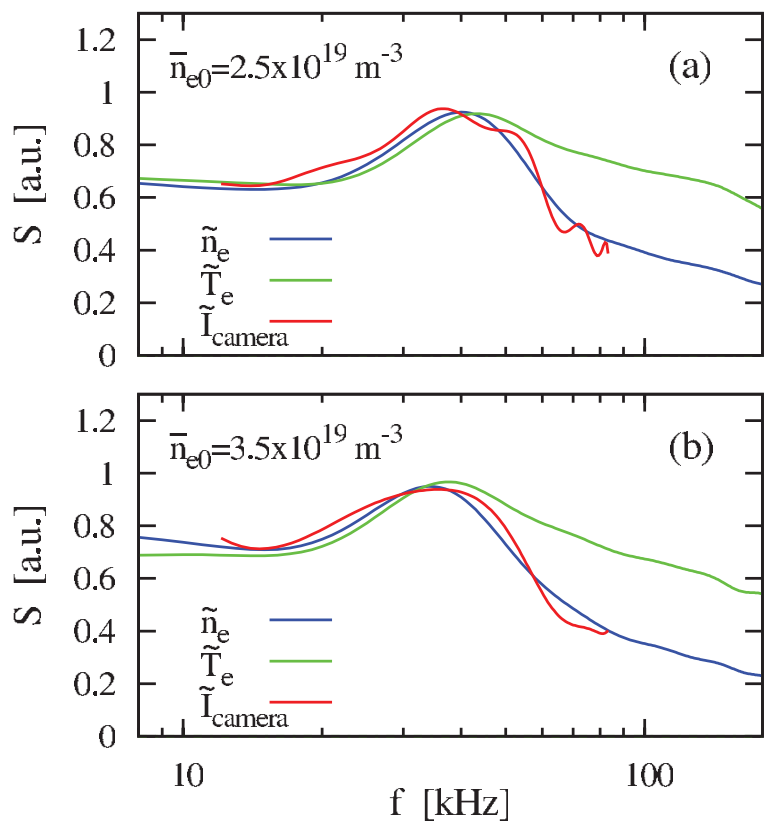

FIG. 9. Comparison of the frequency spectra of $\tilde{n}_{e}$ (blue), $\tilde{T}_{e}$ (green), and $\tilde{I}_{\text {camera }}$ (red). Each result is an average of six spectra corresponding to different shots with the same line-averaged density. Figures 9(a) and 9(b) correspond to series of shots with $\bar{n}_{e 0}=2.5 \times 10^{19} \mathrm{~m}^{-3}$ and $\bar{n}_{e 0}=3.5$ $\times 10^{19} \mathrm{~m}^{-3}$, respectively.

high density shots. Meanwhile, in ohmic plasmas high density shots have higher turbulence magnitudes (before approaching the detachment or density limit). This is favourable as far as we are interested in the fluctuating part of the signal.

Based on results of Figures 7 and 8 we can conclude that high density shots are preferential for the operation of the GPI system. On one hand plasma parameters are less perturbed by the gas puff, on other hand the $I_{\text {camera }}$ and fluctuation magnitude are higher, which are favourable for the signal-to-noise ratio of the GPI diagnostic.

The fluctuation properties of the GPI signal depend on both density and temperature fluctuations. In order to gain deeper insight into this issue, we performed additional studies. Figure 9 shows the comparison of powers spectra of $\tilde{n}_{e}$, $\tilde{T}_{e}$, and $\tilde{I}_{\text {camera }}$, measured at $\mathrm{r}=45.5 \mathrm{~cm}$ for two sets of shots (corresponding to $\bar{n}_{e 0}=2.5 \times 10^{19} \mathrm{~m}^{-3}$ (a) and $\bar{n}_{e 0}$ $\left.=3.5 \times 10^{19} \mathrm{~m}^{-3}(\mathrm{~b})\right)$. The spectra of the $\tilde{T}_{e}$ and $\tilde{n}_{e}$ were measured by triple Langmuir probes. In the figure, each curve is an average of spectra over 6 shots under the same $\tilde{n}_{e 0}$. Because in the performed experiment the Langmuir probe and the GPI system have different sampling time ( 2 and $6 \mu$ s, respectively), the corresponding Nyquist frequencies are also different ( 250 and $83.3 \mathrm{kHz}$, respectively). Therefore, the frequency range of the GPI signal in Figure 9 is shorter than that of the Langmuir probe. Also, it has been found that the frequency spectrum of the fast camera contains parasitic low frequency noise which has most of its energy in the range below $10 \mathrm{kHz}$. As such, the frequency spectrum of the GPI signal fluctuations in Figure 9 starts from $10 \mathrm{kHz}$. Therefore, the comparison between the GPI fluctuation spectrum and the Langmuir probe spectrum can only be made in the frequency range between 10 and $83 \mathrm{kHz}$. The remarkable similarity between the $\tilde{I}_{\text {camera }}$ spectrum and $\tilde{n}_{e}$ spectrum indicates that the $\tilde{I}_{\text {camera }}$ is more sensitive to the $\tilde{n}_{e}$ than to $\tilde{T}_{e}$. It is noticed that this phenomenon remains the same regardless the plasma density $\bar{n}_{e 0}$ in Figures 9(a) and 9(b), even though the positions of peaks in two figures are slightly different.

\section{EXPERIMENTAL RESULTS}

Figure 10 shows a series of frames taken during an ohmic deuterium discharge with $\bar{n}_{e 0}=3.0 \times 10^{19}{ }^{-3}, I_{p}=300 \mathrm{kA}$, $B_{T}=2.6 \mathrm{~T}$ and with exposure time of $6 \mu \mathrm{s}$. The radial (TEXTOR minor radius) and vertical (poloidal) scales are indicated in the first frame. The LCFS is indicated by the dashed green line. The radial outward direction in each frame is towards the left and the poloidal electron diamagnetic drift direction is shown by the arrow in the left side of the figure. As the imaging is along the magnetic field line, the vertical vs radial spatial scales of the structures have the order of the poloidal and radial turbulence correlation lengths.

The images have been processed to remove noise sparks from the frames. Each frame of the GPI data has been filtered with the 2D low-pass filter (with the convolution kernel $[3 \times 3])$ to partially suppress the pixel noise and hold main turbulent events. Previous authors ${ }^{1,26}$ have presented their GPI results in terms of the absolute intensity value (scaled from 0 to maximum). In our case, as we are interested only

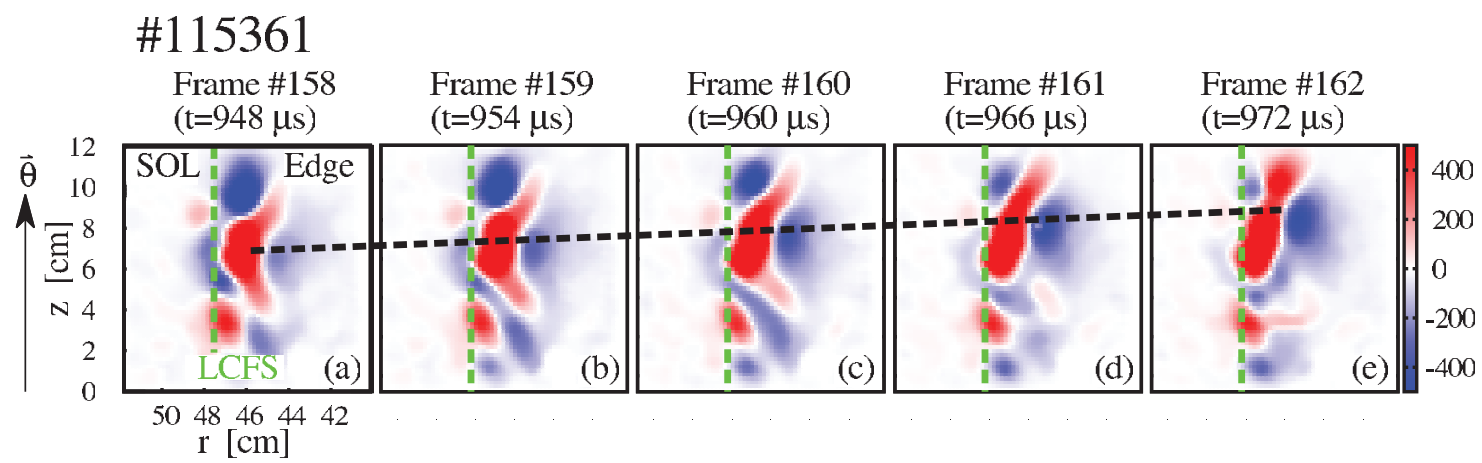

FIG. 10. A set of GPI images taken with an exposure time of $6 \mu$ s during a typical ohmic discharge at TEXTOR $\left(I_{p}=300 \mathrm{kA}, \bar{n}_{e 0}=3 \times 10^{19} \mathrm{~m}^{-3}\right.$, $B_{T}=2.6 \mathrm{~T}$ ). The measurements have been performed in the deuterium $D_{\alpha}$ line. The radial (TEXTOR minor radius) and vertical (poloidal) scales are indicated in the first frame. The black dashed line indicates the poloidal movement of the individual turbulent structure. The green dashed line indicates the position of the separatrix. The radial outward direction is towards the left. 


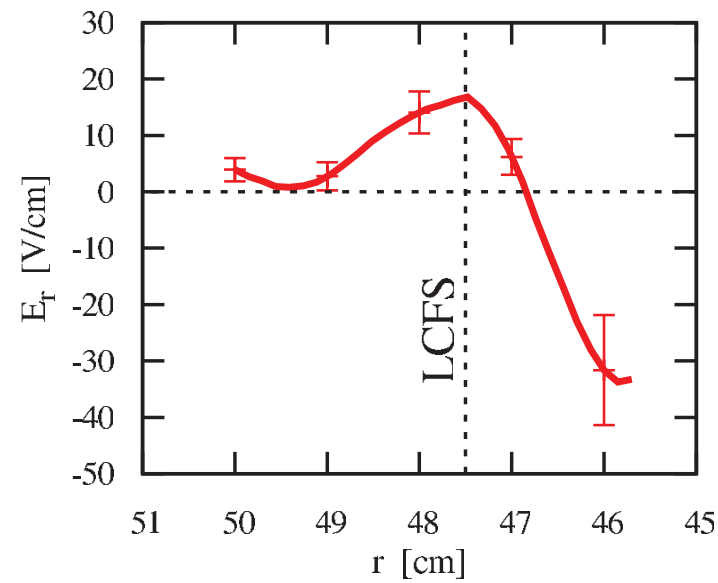

FIG. 11. Radial profile of the mean radial electric field $E_{r}$. The vertical dashed line denotes the LCFS position. The error bars indicate the standard deviation about the mean measured in similar discharges.

in turbulent behaviors, we subtract the time-averaged mean values from the light intensity detected at each pixel for every image. The radial distribution of the emission intensity exhibits a significant radial inhomogeneity as seen from Figures 5(b) and 10. For some applications, such as velocity vector field reconstruction, it might be necessary to highlight far SOL and edge regions and, therefore, compensate such a inhomogeneity. For instance, one can divide the instant value of the fluctuations in each pixel either by the RMS value of fluctuations $\tilde{I}_{\text {camera }}^{R M S}$ or by mean time-averaged value $I_{\text {camera }}$ in that pixel. It has not been performed in Fig. 10 as we prefer to draw readers' eyes directly to the important dynamics of the central structure.

The red color structures in Figure 10 represent edge turbulent eddies. In Figure 10, the typical radial and poloidal dimension of the eddies is $\sim 2 \mathrm{~cm}$ and $6 \mathrm{~cm}$, respectively. The typical moving speed of turbulent eddies in the plasma edge is about $0.3-0.5 \mathrm{~km} / \mathrm{s}$ along the radial and $1-3 \mathrm{~km} / \mathrm{s}$ along the poloidal direction. In the poloidal direction, the eddy moves downward in the SOL (along the ion diamagnetic drift direction) and upward in the plasma edge (along the electron diamagnetic drift direction), following up mainly with the $E_{r} \times B$ drift (will be discussed in the next paragraph). The black dashed line indicates the poloidal movement of the individual turbulent eddy. From the time sequence of Figure 10, we can see that the turbulent eddy is moving poloidally upward at a speed of $V_{\theta} \approx 1 \mathrm{~km} / \mathrm{s}$.

The radial dependence of the $E_{r}$ is depicted in Fig. 11. It was measured using fast reciprocating Langmuir probes. ${ }^{25}$ The equilibrium $E_{r}$ is deduced from the plasma potential $\phi_{p}$ $=\phi_{f l}+2.8 T_{e}$, where $\phi_{f l}$ (floating potential) and $T_{e}$ (electron temperature) are measured by a triple probe. The corresponding $E_{r} \times B$ drift velocity in the plasma edge is $V_{E_{r} \times B}=\frac{E_{r}}{B_{T}}$ $\approx 1.16 \mathrm{~km} / \mathrm{s}$, in good agreement with $V_{\theta}$ from Figure 10 . Detailed inspection on the impact of the mean $E_{r} \times B$ flow shear on tilting and breaking of turbulent eddies has been reported in Ref. 27.

It is interesting to note that in all of our measurements the decay of blob structures appears to be very fast once they move out across the LCFS position. They decay so fast that we never observed any blobby behaviour further than $2-3 \mathrm{~cm}$ outside the LCFS. This result is different from what has been observed in NSTX and C-Mod, where long-standing blobs are propagating far outside the separatrix. This difference can be partially explained by shorter connection length of the magnetic field line to the TEXTOR toroidal (or even poloidal) limiter and consequently more effective dissipation due to fast decay of the parallel particle flux. Furthermore, because of the limiter features, the SOL of TEXTOR usually has lower temperature than diverter machines such as NSTX and C-Mod, which results in less emission intensity in the further region of the SOL.

\section{CONCLUSION}

The GPI diagnostic, ${ }^{1,2}$ assigned to measure 2D effects of the plasma turbulence, has been developed for the TEXTOR tokamak. The in-vessel GPI telescope observes the emission from puffed gas along local (at the puff) magnetic field lines. The GPI telescope is designed to operate in severe TEXTOR conditions and can be used as a prototype for the GPI systems on next generation machines. The results of this work suggest that the local gas puff with the flux $(6-8) \times 10^{19}$ molecules/s, typical for the GPI experiment on TEXTOR, does not influence substantially on local and global plasma performance neither on plasma turbulence properties. Also, it has been shown that the equilibrium/fluctuating emission intensity is more sensitive to the equilibrium/fluctuating electron density than the equilibrium/fluctuating electron temperature, supporting the idea that the GPI is the diagnostic that measures primarily density events. The poloidal propagation velocity, measured with the GPI, follows up mainly with the $E_{r} \times B$ drift. The difference in the blob dynamics in the SOL has also been identified between diverter and limiter tokamaks.

\section{ACKNOWLEDGMENTS}

I. Shesterikov thanks A. Tabula for continuous encouragement. The authors are indebted to S. J. Zweben and R. Maqueda, who gave valuable advices for the development and implementation of the GPI system. The authors would like to thank G. Esser, R. Philips, J. Schruff, H. Reimer for technical assistance on the GPI diagnostic and H. Stoschus, M. Lehnen, S. Brezinsek, O. Schmitz, R. Koslowski, B. Unterberg, T. Lambertz, R. Koch, D. Reiter, and U. Samm for scientific support of this work.

${ }^{1}$ S. J. Zweben, D. P. Stotler et al., Phys. Plasmas 9, 1981 (2002).

${ }^{2}$ R. J. Maqueda, G. A. Wurden et al., Rev. Sci. Instrum. 74, 2020 (2003).

${ }^{3}$ Y. Xu et al., Plasma Phys. Controlled Fusion 47, 1841 (2005).

${ }^{4}$ N. Mahdizadeh et al., Phys. Plasmas 11, 3932 (2004).

${ }^{5}$ B. LaBombard et al., Nucl. Fusion 40, 2041 (2000).

${ }^{6} \mathrm{M}$. Umansky et al., Phys. Plasmas 5, 3373 (1998).

${ }^{7}$ T. A. Carter et al., Phys. Plasmas 16, 012304 (2009).

${ }^{8}$ P. Manz et al., Plasma Phys. Controlled Fusion 50, 035008 (2008).

${ }^{9}$ I. Furno et al., Phys. Plasmas 15, 055903 (2008).

${ }^{10}$ S. Zoletnik et al., Rev. Sci. Instrum. 76, 073504 (2005).

${ }^{11}$ G. R. McKee et al., Phys. Plasmas 10, 1712 (2003).

${ }^{12}$ G. Y. Antar et al., Phys. Plasmas 14, 022301 (2007)

${ }^{13}$ S. A. Bozhenkov et al., Nucl. Fusion 51, 083033 (2011). 
${ }^{14}$ Ch. P. Ritz et al., Rev. Sci. Instrum. 59, 1739 (1988).

${ }^{15}$ S. J. Zweben and S. S. Medley, Phys. Fluids B 1, 2058 (1989).

${ }^{16}$ R. J. Maqueda et al., Rev. Sci. Instrum. 72, 931 (2001).

${ }^{17}$ H.-J. Kunze, Introduction to Plasma Spectroscopy (Springer, Berlin, 2009).

${ }^{18}$ T. Fujimoto, Plasma Spectroscopy (Springer, Berlin, 2004).

${ }^{19}$ J. D. Hey et al., J. Phys. B 32, 3555 (1999).

${ }^{20}$ J. D. Hey et al., J. Phys. B 37, 2543 (2004).

${ }^{21}$ R. Janev, Elementary Processes in Hydrogen-Helium Plasmas (Springer, Berlin, 1987).
${ }^{22} \mathrm{~S}$. Brezinsek, "Study of atomic and molecular hydrogen in front of a graphite surface in a high-temperature edge plasma," $\mathrm{Ph} . \mathrm{D}$. dissertation (Heinrich Heine University Dusseldorf, 2001).

${ }^{23}$ S. Brezinsek et al., Plasma Phys. Controlled Fusion 47, 615 (2005).

${ }^{24}$ E. Hintz and B. Schweer, Plasma Phys. Controlled Fusion 37, A87 (1995).

${ }^{25}$ Y. Xu et al., Nucl. Fusion 47, 1696 (2007).

${ }^{26}$ S. J. Zweben et al., Nucl. Fusion 44, 134 (2004).

${ }^{27}$ I. Shesterikov, Y. Xu et al., Nucl. Fusion 52, 042004 (2012).

${ }^{28}$ O. Grulke et al., Phys. Plasmas 13, 012306 (2006). 